\title{
Assessment of Micro-Albuminuria and Renal Artery Resistance Index in Psoriatic Patients
}

\author{
N.E.Sorour ${ }^{1}$, N.A.AbdulHafeez ${ }^{2}$, E.M.Akl ${ }^{1}$, H.M.Khater ${ }^{3}$ and A.H.El-Shahat ${ }^{1}$ \\ ${ }^{1}$ Dermatology,Venereology and Andrology Dept., Faculty of Medicine, Benha Univ., Benha, Egypt \\ ${ }^{2}$ Clinical Pathology and Immunology Dept., Faculty of Medicine, Benha Univ., Benha, Egypt \\ ${ }^{3}$ Diagnostic Radiology Dept., Faculty of Medicine, Benha Univ., Benha, Egypt \\ E-Mail: amynhsalm0@gmail.com
}

\section{Abstract}

\begin{abstract}
Psoriasis is a common chronic inflammatory skin disease with a spectrum of clinical phenotypes and results from the interplay of genetic, environmental, and immunological factors. Micro albuminuria is considered to be maker of glomular disease and can be used to predict diabetic or hypertensive nephropathy. The aim of the study was to evaluate microalbuminuria and renal artery resistance index in psoriatic patients as an early diagnostic marker of kidney affection. Methods: This was a cross sectional caselcontrol study, included 84 subjects classified into two groups: Patient group: 50 patients complaining of psoriasis. Control group: 34 apparently healthy of matched age and sex as control grouP.Each patient was subjected to full history taking, and complete clinical examination, Clinical assessment of psoriatic lesions is done to to determine the distribution, clinical variants and the extent of psoriasis. Assessment of psoriasis grade was done using PASI score. All studied subjects were tested for: Measuring microalbumuria in urine by ELISA. Results and conclusion: On comparing both groups of patients and controls in this study there was a significant increase of the mean level of microalbuminuria in psoriatic patients compared with controls. There was a positive correlation between the level of microalbuminuria and severity of psoriasis (PASI score) with a significant value. Microalbuminuria and renal artery resistance were elevated in psoriatic patients and has positive correlation with disease severity and may have a role in disease pathophysiology.
\end{abstract}

Keywords: Micro-Albuminuria, Renal, Psoriasis, Resistance Index.

\section{Introduction}

Psoriasis is a multisystem disease with predominantly skin and joint manifestations affecting approximately $2 \%$ of the population. The major manifestation of psoriasis is chronic inflammation of the skin. It is characterized by disfiguring, scaling and erythematous plaques that may be painful or often severely pruritic and may cause significant quality of life issued [1].

Psoriasis is generally thought to be a genetic disease that is triggered by environmental factors. In twin studies, identical twins three times more likely to be affected compared to non-identical twins. This suggests that genetic factors predispose to psoriasis. Symptoms often worsen during winter and with certain medications, such as beta blockers or NSAIDs. Infections and psychological stress can also play a role. Psoriasis is not contagious. The underlying mechanism involves the immune system reacting to skin cells [2].

In a study done by Ryan et al., [3], patients with psoriasis had an increased prevelance of pathological microalbuminuria compared with controls. The positive correlation between the prevalence of pathological albuminuria and psoriasis severity may indicate a subclinical renal dysfunction in patients with psoriasis.

Micro albuminuria is considered to be maker of glomular disease and can be used to predict diabetic or hypertensive nephropathy. Doppler derived renal resistive index (RRI) has been used for years in variety of clinical seating such as: evaluation of progression risk in chronic kidney disease [4].

Clinical and experimental evidence indicates that an increased RRI in patients with primary hypertension not only reflects changes in intra renal perfusion, but it is also associated with systemic hemodynamics and atherosclerosis, and may provide useful prognostic information and possibly have therapeutic implications [5].

The aim of the study was to evaluate microalbuminuria and renal artery resistance index in psoriatic patients as an early diagnostic marker of kidney affection.

\section{Patients and methods}

This was a cross sectional caselcontrol study, included 84 subjects classified into two groups:

I-Patient group: 50 patients complaining of psoriasis.

II-Control group: 34 apparently healthy of matched age and sex as control group.

Patients were recurrited from the outpatient clinic of Dermatology and Andrology Department of Benha University Hospitals between October 2018 and February 2019.

The study was approved by the local ethic committee on human research of Benha Faculty of Medicine. Informed consent was obtained from each individual before being enrolled in the study.

\subsection{Inclusion criteria}

This study includes psoriatic patients which are:

- Male and female patients suffering from plaque psoriasis more than 6 months durations.

- Ages more than 18 years old.

- PASI score more than 8 .

- Patients stopped treatment one month before beginning of the procedure. 


\subsection{Exclusion criteria}

Patients with any of the following were excluded:

- Patients with other autoimmune or allergic diseases to avoid false positive results.

- Patients with hepatic diseases to avoid false positive results.

- Patients with unstable psoriasis (pustular, erythrodermic).

- Pregnant patients.

- Patients who refuse to participate in the study.

- Patients with other dermatological diseases.

Each patient was subjected to full history taking, and complete clinical examination, Clinical assessment of psoriatic lesions is done to to determine the distribution, clinical variants and the extent of psoriasis. Assessment of psoriasis grade was done using PASI score: Psoriasis Area and Severity Index (PASI) score is a tool used to measure the severity and extent of psoriasis. It takes a few minutes and experience to calculate it accurately.

All studied subjects were tested for: Measuring microalbumuria in urine by commercial ELISA kit (CarlZeisis-Strabe49-5155129 Mainz-Germany). And Measuring renal artery resistance index by Doppler ultrasound.

\subsection{Statistical analysis}

The clinical data were recorded on a report form. These data were tabulated and analysed using the computer program SPSS (Statistical package for social science) version 20 to obtain: Descriptive statistics were calculated for the data in the form of: Mean and standard deviation ( $\pm S D$ ). Median and inter-quartile range (IQR) for quantitative data. Frequency and distribution for qualitative data. Analytical statistics : In the statistical comparison between the different groups, the significance of difference was tested using one of the following tests. Student's $t$-test:- Used to compare mean of two groups of quantitative data. ANOVA test ( $F$ value):-Used to compare mean of more than two groups of quantitative data. Inter-group comparison of categorical data was performed by using chi square test $\left(X^{2}\right.$-value) and fisher exact test (FET).

\section{Results}

The current study included fifty psoriatic patients; 38 males $76.0 \%$ and 12 females $24.0 \%$. Their ages ranged from 22-75 years with a mean age of $49.86 \pm 11.22$ years. Thirty Four clinically free individuals served as a control group; 24 males $70.6 \%$ and 10 females $29.4 \%$. Their ages ranged from 31-66 years with a mean age of $46.71 \pm 16.7$ years. Smoking habit found in $17(34 \%)$ and $14(41.2 \%)$ in case and control group respectively.

Comparison between patients and control showed no significant difference regarding sex , age, BMI, smoking respectively ( $\mathrm{p}=0.58,0.30,0.028,0.50$ Resp) Table (1).

Table (1) Personal characteristics of the studied groups.

\begin{tabular}{|c|c|c|c|c|c|c|}
\hline & \multicolumn{2}{|c|}{ patients group (50) } & \multicolumn{2}{|c|}{ Control group (34) } & \multirow[t]{2}{*}{ Statistical test } & \multirow[t]{2}{*}{$P$ value } \\
\hline & No & $\%$ & No & $\%$ & & \\
\hline Gender & 38 & & & & & \\
\hline Male & 12 & 76.0 & 24 & 70.6 & $X^{2}=0.31$ & 0.58 \\
\hline Female & & 24.0 & 10 & 29.4 & & \\
\hline Mean \pm SD & \multicolumn{2}{|c|}{$49.86 \pm 11.22$} & \multicolumn{2}{|c|}{$46.71 \pm 16.7$} & St $t=1.04$ & 0.30 \\
\hline BMI & \multicolumn{2}{|c|}{$29.42 \pm 2.52$} & \multicolumn{2}{|c|}{$27.97 \pm 3.43$} & St $\mathrm{t}=2.23$ & 0.028 \\
\hline \multicolumn{7}{|l|}{ Mean \pm SD } \\
\hline Yes & 17 & 34.0 & 14 & 41.2 & $X^{2}=0.45$ & 0.50 \\
\hline No & 33 & 66.0 & 20 & 58.8 & & \\
\hline
\end{tabular}

St $\mathrm{t}=$ student $\mathrm{t}$ test, $\mathrm{x}^{2}=$ chi square test.

Results of the study showed that 8 patients $(16.0 \%)$ had positive family history for psoriasis. The mean duration of psoriasis was $18.96 \pm 8.9$ years and the mean PASI score was $10.27 \pm 2.04$ Table (2).

Table (2) Clinical characteristics among studied groups.

\begin{tabular}{lcc}
\hline & \multicolumn{2}{c}{ Patients group (50) } \\
\cline { 2 - 3 } & No & $\%$ \\
\hline Duration (years) & $18.96 \pm 8.9(2-40)$ \\
Mean \pm SD (range) & & \\
Family history & & \\
Yes & 8 & 16.0 \\
No & 42 & 84.0 \\
PASI Score & $10.27 \pm 2.04(8-14.1)$ \\
Mean \pm SD (range) & \multicolumn{2}{c}{} \\
\hline
\end{tabular}


The mean microalbuminuria levels was signficantly higher in psoriatic patients (18.08) than control group
(11.77) with a significant value $(\mathrm{p}=0.001)$ Table (3).

Table (3) Microalbuminurea level of the studied groups.

\begin{tabular}{lcccc}
\hline & Patients group (50) & Control group (34) & Statistical test & P value \\
\hline $\begin{array}{l}\text { Microalbuminurea } \\
\text { Mean } \pm \text { SD }\end{array}$ & $23.04 \pm 18.08$ & $11.77 \pm 7.17$ & St $t=3.45$ & $0.001^{* *}$ \\
\hline
\end{tabular}

St $\mathrm{t}$ test $=$ student test.

Mean microalbuminurea level in female patients was higher than male patients without a significant value $(\mathrm{p}=0.14)$. The mean microalbuminurea level in patients with +ve family history was higher than patients with -ve
Family history without significant value $(\mathrm{p}=0.60)$. Mean microalbuminurea level in smoking patients was higher than non-smoking patients with a highly significant value $(\mathrm{p}=<0.001 * *)$ Table (4).

Table (4) Mean Microalbuminuria differences of patients group according to gender, family history and smoking.

\begin{tabular}{|c|c|c|c|c|c|}
\hline \multirow[t]{2}{*}{ Patients group (50) } & \multirow[t]{2}{*}{ No } & \multicolumn{2}{|c|}{ Microalbumin } & \multirow[t]{2}{*}{ Statistical test (t) } & \multirow[t]{2}{*}{ P value } \\
\hline & & Mean & \pm SD & & \\
\hline \multicolumn{6}{|l|}{ Gender } \\
\hline Male & 38 & 20.91 & 17.03 & 1.5 & 0.14 \\
\hline Female & 12 & 29.79 & 20.38 & & \\
\hline \multicolumn{6}{|l|}{ Family history } \\
\hline Present & 8 & 26.14 & 16.28 & 0.52 & 0.60 \\
\hline Absent & 42 & 22.45 & 18.52 & & \\
\hline \multicolumn{6}{|l|}{ Smoking } \\
\hline Yes & 17 & 37.01 & 23.12 & 4.69 & $<0.001 * *$ \\
\hline No & 33 & 15.85 & 8.73 & & \\
\hline
\end{tabular}

St $\mathrm{t}$ test $=$ student $\mathrm{t}$ test.

Mean microalbuminurea level in severe PASI score patients was higher than moderate PASI score patients with a highly significant value $(\mathrm{p}=0.007 * *)$ Fig $(1)$.

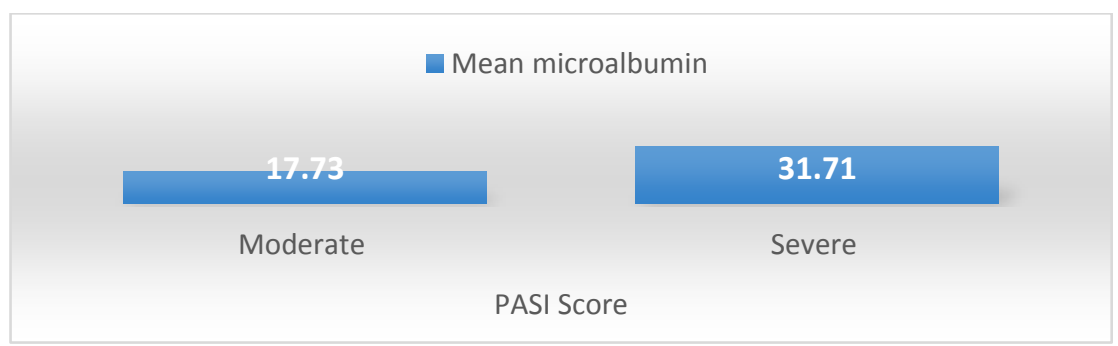

Fig (1) Receiver operating characteristics (ROC) analysis of microalbuminurea for discrimination between patients and control.

In psoriatic patients, there was positive correlations between microalbuminuria level and PASI score, RT renal artery RI, LT ranal artery RI with a significant value as $\mathrm{p}$ value $(0.001,0.021 \& 0.036)$ respectively Table (5).

Table (5) Correlation between microalbuminurea and other quantitative variables.

\begin{tabular}{lcc}
\hline Micro albumin & Correlation coefficient & P value \\
\hline Age & -0.19 & 0.18 \\
BMI & -0.05 & 0.72 \\
Age of onset & -0.16 & 0.28 \\
Duration & -0.07 & 0.65 \\
PASI score & 0.495 & $<0.001^{* *}$ \\
Rt renal artery RI & 0.352 & $0.021^{*}$ \\
Lt renal artery RI & 0.297 & $0.036^{*}$ \\
\hline
\end{tabular}


Receiver operating characteristics (ROC) analysis of microalbuminurea for discrimination between patients and control : Results of the study showed that microalbuminurea had positive sensitivity followed by specificity with a significant value $(\mathrm{P}$ value $<0.05)$ Fig (2).

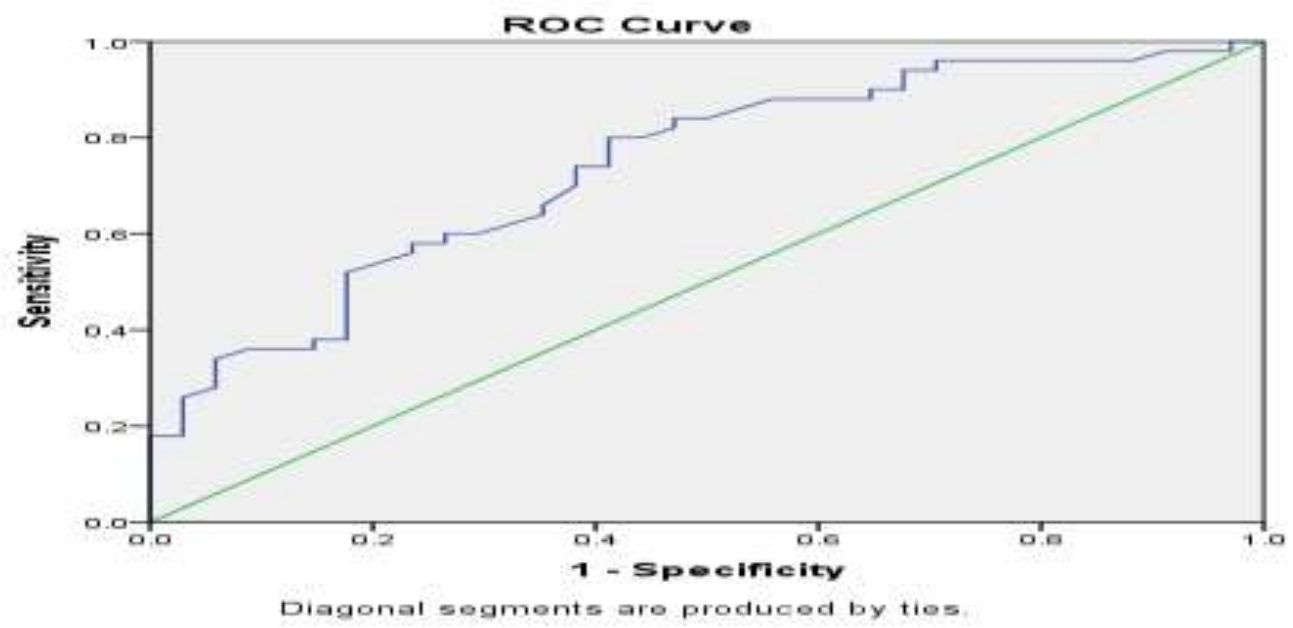

Fig (2) Receiver operating characteristics (ROC) analysis of microalbuminurea for discrimination between patients and control.

\section{Discussion}

Psoriasis is a chronic, inflammatory, immunemediated skin condition that is characterized by welldemarcated, erythematous plaques with silver scales, which can be associated with potential symptoms of pruritus, pain, skin tightness, bleeding, and flaking [6].

In the current study, there was no significant difference between psoriatic patients and control groups regarding sex.

Zalewska et al. [7], found in the clinical approach, that psoriasis as a chronic disease is defined as diseases of a long duration, but of milder course than acute illnesses.These results agreed with the current study, which reported that psoriatic patients suffered from the disease for long period of time, which denotes that psoriasis is a chronic disease.

In the current study, there was highly significant difference between course of psoriasis and microalbuminuria. these results showed that psoriatic patients with progressive course have higher microalbuminuria level than psoriatic patients with intermittent course.

In the current study, there was nonsignificant value between patients with positive family history and negative family history, although microalbuminuria level was higher in patients with positive family history than those patients with negative family history. These results were in agreement with those of [8], who found nonsignificant difference between patients and control subjects regarding family history.

The obtained results demonstrated that only $16.0 \%$ of psoriatic patients have positive family history. Psoriasis has been sub classified according to age of onset. Early onset psoriasis (also referred to as type I) has onset before the age of 40 years, with peak onset at 16-22 years of age, and comprises $70 \%$ of all psoriatic. Late-onset psoriasis, also termed type II psoriasis, shows onset at or after age 40 years, with a peak age of onset between 57 and 60 years. A distinct pattern of HLA association has been reported. Hence, with early onset psoriasis, which also displays a strong family history, a strong association with class I HLA alleles, and specifically HLA-C*06, is observed. In contrast, type II psoriasis is more sporadic and rarely familial and its genetic background is unclear [9]. Results of Kim et al. [10], demonstrated increased severity of skin lesions and nail affection in early onset psoriasis with strong family history of psoriasis.

Central obesity, hypertension, hyperglycemia, and dyslipidemia are the main components of metabolic syndrome or syndrome X [11]. Study of Gisondi et al. [12] conducted that the prevalence of metabolic syndrome in patients with psoriasis ranges from $20 \%$ to $50 \%$, with a risk of having metabolic syndrome is at least double in psoriatic patients compared with non-psoriatic control individuals. The association between psoriasis and metabolic syndrome has important clinical implications and the strength of these associations has been repeatedly confirmed by several observational studies.Thus improve the strong association between psoriasis with hyperlipidemia and hyperglycemia which are components of metabolic syndrome.

In the current study, there was nonsignificant value between males and females regarding microalbuminuria level, although micro albuminuria level was higher in females than males. These results agreed with the previous finding of Kaur et al, [13] reported no sex predilection among study patients.

The current study showed that there was statistically highly significant positive correlation found between microalbuminuria and PASI score.

So, severe cases of psoriasis can be associated with high level of micro albuminuria, therefore micro albuminuria can be a biomarker of psoriasis severity. 
In the current study, there was highly significant difference between smoking psoriatic patients and non smoking psoriatic patients. these results showed that smoking patients have higher microalbuminuria level than non smoking patients.

In the current study, there was non significant difference between microalbuminuria level and nail affection, although micro albuminuria level was in patients without nail affection than in patients with nail affection.

In the current study, there was a highly significant difference in left renal artery resistance index value in psoriatic patients than psoriatic patients. and there was a significant difference in right renal artery resistance value in psoriatic patients than non psoriatic patients.

In the current study, there was nonsignificant difference between microalbuminuria level and history of arthritis, history of previous of treatment and history of systemic disease.

Results of the current study showed that microalbuminuria levels and renal artery resistance index were significantly higher in psoriatic patients when compared to controls.

\section{Conclusion}

Microalbuminuria and renal artery resistance were elevated in psoriatic patients and has positive correlation with disease severity and may have a role in disease pathophysiology.

\section{References}

[1] A. Menter ."Guidelines of care for the management of psoriasis and psoriatic arthritis: Section 1 . Overview of psoriasis and guidelines of care for the treatment of psoriasis with biologics," J. Am. Acad. Dermatol.Vol.58, PP.826-850, 2008.

[2] A. M. Bowcock and J. G. Krueger. Getting under the skin: the immunogenetics of psoriasis," Nat. Rev. Immunol.Vol.5, PP.699-711, 2005.

[3] C. Ryan . "Research gaps in psoriasis: opportunities for future studies," J. Am. Acad. Dermatol.Vol.70, PP.146-167, 2014.
[4] J. Radermacher and H. Haller. "The role of the intrarenal resistive index in kidney transplantation." Mass Medical SocVol.1, PP.56-71, 2013.

[5] F. Viazzi, G. Leoncini, L. E. Derchi, and R. Pontremoli, "Ultrasound Doppler renal resistive index: a useful tool for the management of the hypertensive patient," J. Hypertens.Vol.32, PP.149, 2014.

[6] N. J. Korman. Management of psoriasis as a systemic disease: what is the evidence?," $\mathrm{Br}$. J. Dermatol.Vol.182, PP.840-848, 2020.

[7] A. Zalewska, J. Miniszewska, J. Chodkiewicz, and J. Narbutt, "Acceptance of chronic illness in psoriasis vulgaris patients," J. Eur. Acad. Dermatology Venereol.Vol.21, PP.235-242, 2007.

[8] Y. Zheng . Interleukin-22, a TH 17 cytokine, mediates IL-23-induced dermal inflammation and acanthosis," NatureVol.445, PP.648-651, 2007.

[9] R. Queiro, P.Tejón, S. Alonso, and P.Coto, “Age at disease onset: a key factor for understanding psoriatic disease," RheumatologyVol.53, PP.11781185, 2014.

[10] W. B. Kim, D. Jerome, and J. Yeung, "Diagnosis and management of psoriasis," Can. Fam. PhysicianVol.63, PP.278-285, 2017.

[11] J.-P.Després ."Abdominal obesity and the metabolic syndrome: contribution to global cardiometabolic risk," Arterioscler. Thromb. Vasc. Biol.Vol.28, PP.1039-1049, 2008.

[12] P.Gisondi, A. C. Fostini, I. Fossà, G. Girolomoni, and G. Targher, "Psoriasis and the metabolic syndrome," Clin. Dermatol.Vol.36, PP.21-28, 2018.

[13] I. Kaur, V. Gandhi, A. Raizada, S. N. Bhattacharya, A. K. Tripathi, and D. Jakhar, "Psoriatic nephropathy and its correlation with hs-CRP: A case control study," Indian Dermatol. Online J.Vol.11, P.29, 2020. 